\title{
A Mobile Table for the Communication in IPv4/v6 Mixed Networks
}

\author{
Zheng Xiang \\ College of Medical Information Engineering \\ Guangdong Pharmaceutical College \\ Guangzhou, China
}

\author{
Zhengming $\mathrm{Ma}$ \\ College of Information Science and Technology \\ Sun Yat-sen University \\ Guangzhou, China
}

\begin{abstract}
IETF has specified Mobile IPv4 and Mobile IPv6 in RFC3344 and RFC3775 respectively, but not yet discussed Mobile IPv4/v6 in any published RFC. This paper proposes a scheme to solve one of Mobile IPv4/v6 problems which Home Agent (HA) is located in IPv4 network, Correspondent Node (CN) is located in IPv6 network, while MN moves within IPv4 network. In the solution, a gateway called Mobile IPv4/v6 translation gateway (MIPv4/v6-TG) is introduced to bridge between IPv4 network and IPv6 network, which is made up of a traditional NAT-PT gateway and a Mobile IP application level gateway (MIP-ALG) built upon the NAT-PT gateway. MIP-ALG maintains a MIP table, a data structure, which is formed by entries. We use the MIP table to realize the communication between the IPv4 entities and the IPv6 entities. The creation, usage and update processes of MIP table are described in this paper. Through the experiment we find it can realize the basic communication in IPv4/v6 mixed network. The measurement of performance test proves the solution's efficiency. And it can work compatibly with RFC3344 and RFC3775.
\end{abstract}

IndexTerms-MobileIP(MIP), NAT-PT, Mobile IP application level gateway (MIP-ALG), Mobile IPv4/v6 translation gateway (MIPv4/v6-TG), MIP Table

\section{INTRODUCTION}

IETF specified the latest version of Mobile IPv4 [1] and Mobile IPv6[2] in RFC3344 and RFC3775 respectively. These two protocols work very well in pure IPv4 network and pure IPv6 network. However, both of them can not be directly applied to IPv4/v6 mixed networks. As the current network evolves gradually from IPv4 to IPv6, more and more mobile nodes need to roam in IPv4/v6 mixed networks. So we need to develop a transition scheme to support Mobile IP in IPv4/v6 mixed networks.

Currently, three transition ways are recommended by IETF, namely, dual stacks (RFC4213) [3], tunneling (RFC3053) [4], and NAT-PT (RFC2766) [5][6]. There have been a lot of studies on Mobile IP in IPv4/v6 mixed networks, many of which are based on NAT-PT. An Internet Draft published by IETF [7] presents a solution to a situation of Mobile IPv4/v6, in which HA and MN are in IPv6 network, CN is in IPv4 network and NAT-PT gateway is located between IPv4 network and IPv6 network. It is proposed in this draft that NAT-PT do all of MIPv6 functionalilties on behalf of CN.

This paper proposes a scheme to solve one of Mobile IPv4/v6 problems which Home Agent (HA) is located in IPv4

This work is supported in part by The Medium and Small Enterprise Technology Innovation Fund of The Ministry of Science and Technology (No. 09C26214402150), China. network, Correspondent Node (CN) is located in IPv6 network, while MN moves within IPv4 network. The solution is based on Mobile IPv4/v6 Translation Gateway (MIPv4/v6-TG) which is made up of NAT-PT and a Mobile IP-Application Level Gateway (MIP-ALG). And we will introduce the data structure, MIP table, which is maintained by MIP-ALG, and describe in details how to create, use and update MIP table entries. We use the MIP table to realize the communication between the Mobile IPv4 entities and the Mobile IPv6 entities.

\section{TERMINOLOGY}

The terms frequently used in this paper are listed in Table1.In addition, "v4" and "v6" are used to indicate IPv4 and IPv6, respectively. For example, HAv6 stands for HA that is located in IPv6 domain and CNAv6 the IPv6 address of CN. If an IPv4 address is marked with a “\#”, it stands for an address from the address pool of NAT-PT gateway and is used to map an IPv6 address. For example, HAAv4\# is an address used by NAT-PT gateway to map HAAv6. Packets with HAAv4\# as their destination addresses will be routed to NATPT gateway and delivered to HAv6. If an IPv6 address is marked with a “*”, it means that this address is made up of a 96-bit NAT-PT prefix and an IPv4 address. For example, CNAv6* is made up of a NAT-PT prefix and CNAv4. Packets with CNAv6* as their destination addresses will be routed to NAT-PT gateway and delivered to CNv4.

TABLE I. TERMS THAT ARE FREQUENTLY USED

\begin{tabular}{cccc}
\hline Terms & Meaning & Terms & Meaning \\
\hline MIP & Mobile IP & CNA & Correspondent Node Address \\
HA & Home Agent & BU & Binding Update \\
CN & Mobile Node & BA & Binding Acknowledge \\
HAA & Home Agent Address & CoTI & Care-of Test Init \\
HoA & Home Address & HoT & Home Test \\
CoA & Care-of Address & CoT & Care-of Test \\
\hline
\end{tabular}




\section{MIP TABLE}

\section{A. $M I P v 4 / v 6-T G$}

MIPv4/v6-TG is made up of NAT-PT and MIP-ALG. On IPv6 network side, MIPv4/v6-TG acts as one of the Mobile IPv6 entities to combine with other MIPv6 entities inside the IPv6 network to form a complete MIPv6 model described in RFC3775. Inside the IPv6 network, the registration process and communication process can be performed as specified in RFC3775. Similarly, on IPv4 network side, MIPv4/v6-TG acts as one of the Mobile IPv4 entities to combine with other MIPv4 entities inside the IPv4 network to form a complete MIPv4 model described in RFC3344. Inside the IPv4 network, the registration process and communication process can be performed as specified in RFC3344.

As mentioned above, MIPv4/v6-TG will act as different MIP entities according to different combinations of the IP versions of $\mathrm{HA}, \mathrm{MN}$ and $\mathrm{CN}$. However, it can not decide which role it should act, if it does not know the IP versions of the three entities when MIPv4/v6-TG intercepts a MIP-related message or datagram. Therefore, MIPv4/v6-TG should keep the information of the IP versions of the three entities and the bindings that are used when it acts as a particular MIP entity. In our solution, we introduce a new data structure called MIP table to solve this problem. We use 3-bit binary numbers to indicate the location where $\mathrm{MN}, \mathrm{HA}$ and $\mathrm{CN}$ sit. If the communication entity location in the IPv6 network we record it as 1 , otherwise we record it as 0 .

The three MIP entities have eight kinds of IP version combinations, from 000 to 111 . At any time, there may be many MIP sessions, which correspond to the same scenario and thus use the same kind of MIP table entry. MIPv4/v6-TG should be able to distinguish them so that the messages and datagrams can be sent to the right place. Therefore, each MIP table entry has four kinds of entrances: MIPv6 Message Entrance, MIPv6 Datagram Entrance, MIPv4 Message Entrance and MIPv4 Datagram Entrance. We will introduce the MIP table in the following.

\section{B. MIP table} fields.

An MIP table entry should, typically, have the following

Type: A three-bit field indicating the IP versions of HA, $\mathrm{MN}$ and $\mathrm{CN}$ respectively. For each bit, a value of 1 indicates the MIP entity is located in IPv6 network, while a value of 0 indicates the MIP entity is located in IPv4 network. Type value varies from 000 to 111 .

MIPv6 Message Entrance: A 128-bit field through which a particular MIP table entry can be found and accessed when a MIPv6 message is intercepted by MIPv4/v6-TG. Usually, this field is set to the IPv6 home address. All MIPv6 messages contain IPv6 home addresses. When a MIPv6 message is intercepted by MIPv4/v6-TG, MIPv4/v6-TG takes out the IPv6 home address from the intercepted message and uses it as an index to search the MIP table. In this process, MIPv4/v6-TG compares the IPv6 message entrance field of each entry with the IPv6 home address. If an entry is found, MIPv4/v6-TG will use the information recorded in the entry to process the intercepted message. If no entry is found, MIPv4/v6-TG will create a new entry.

MIPv6 Datagram Entrance: A 128-bit field through which a particular entry can be found and accessed when a MIPv6 datagram is intercepted by MIPv4/v6-TG. If an intercepted packet is not a MIPv6 message, MIPv4/v6-TG will take out its destination address and use the address as an index to search the MIP table. In this process, MIPv4/v6-TG compares the IPv6 datagram entrance filed of each entry with the address. If an entry is found, the intercepted packet is a MIPv6 datagram, and will be processed based on the information recorded in the entry. If no entry is found, the intercepted packet is not a MIPv6 datagram.

MIPv4 Message Entrance: A 32-bit field through which a particular entry can be found and accessed when a MIPv4 message is intercepted by MIPv4/v6-TG. On intercepting a MIPv4 message, MIPv4/v6-TG takes out its destination address and uses the address as an index to search for the corresponding MIP table entry. It is based on the MIP table entry that MIPv4/v6-TG processes the intercepted MIPv4 message.

MIPv4 Datagram Entrance: A 32-bit field through which a particular entry can be found and accessed when a MIPv4 datagram is intercepted by MIPv4/v6-TG. If an intercepted packet is not a MIPv4 message, MIPv4/v6-TG will take out its destination address and use the address as an index to search the MIP table. In this process, MIPv4/v6-TG compares the IPv4 datagram entrance filed of each entry with the address. If an entry is found, the intercepted packet is a MIPv4 datagram, and will be processed based on the information recorded in the entry. If no entry is found, the intercepted packet is not a MIPv4 datagram.

Cached Bindings: Bindings of home address and care-of address that are used by MIPv4/v6-TG when it acts as a particular MIP entity. Bindings may be of IPv4 form or of IPv6 form. Entries may have no binding, one binding, or two bindings. This depends on the types of the entries.

Source Port: A 16-bit field that records the source port value of Registration Request message, extended Registration Request message, or Agent Request message. All of these three messages are sent through UDP ports. When MIPv4/v6TG intercepts such kind of message, it fills this field with the source port value. This field is used as the destination port when MIPv4/v6-TG sends back a reply later.

Destination Port: A 16-bit field that records the destination port value of Registration Request message, extended Registration Request message, or Agent Request message. It will be uses as the source port when MIPv4/v6-TG sends back a reply later.

State: A 1-bit field indicating whether the entry is completed or not. There are two possible states for each entry: 1 (finished) and 0 (unfinished). An unfinished state indicates that the entry is now being created or updated. Entries with an unfinished state can not be used by MIPv4/v6-TG as a guide to process MIP datagrams. 
Lifetime: A 16-bit field indicating entry lifetime. The meaning of this field depends on the value of the State field. When the State is 0 (unfinished), Lifetime means the entry should be completed before the time expires. When the State is 1 (finished), Lifetime means the number of seconds left before the entry is considered expired. In either case, when this filed becomes $0, \mathrm{MIPv} 4 / \mathrm{v6}$-TG will delete the entry.

\section{APPLICATION EXAMPLE}

We will solve one of Mobile IPv4/v6 problems which Home Agent (HA) is located in IPv4 network, Correspondent Node (CN) is located in IPv6 network, while MN moves within IPv4 network. And we will describe the creation, update and usage of type 001 MIP table entries as an example in the following.

\section{A. The create of Type 001 MIP Table Entries}

Agent Request message is used only when HA is located in IPv4 network, CN is located in IPv6 network, and MN has just moved from IPv6 network to IPv4 network. Since the IP version of $\mathrm{MN}$ has changed from IPv6 to IPv4. The original entry becomes invalid and a new entry should be created. The creation process of the 001 type MIP table entries is shown in Fig.1.

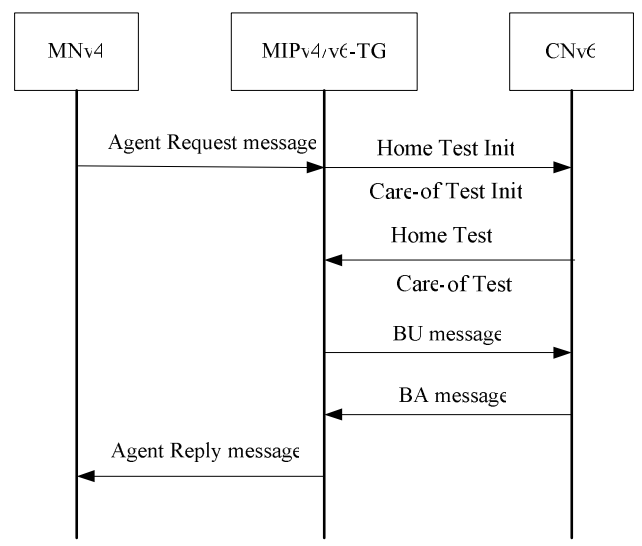

Figure 1. The creation process of the 001 type MIP table entries

The concrete steps as follows:

(1) MIPv4/v6-TG intercepts an Agent Request message and delivers it to MIP-ALG for further processing. Here, MIPv4/v6-TG uses the intercepting mechanism described above to intercept and recognize the Agent Request message.

(2) On receiving the Agent Request message, MIP-ALG can infer that HA is located in IPv4 network, CN is located IPv6 network and MN has just moved from IPv6 network to IPv4 network, as only in this scenario can MIPv4/v6-TG receive an Agent Request message. Therefore, a new entry with the Type value 001 (binary) is created.

MIP-ALG sets the field of MIPv6 Message Entrance to HoAv6*, MIPv6 Datagram Entrance to CoAv6*, MIPv4 Datagram Entrance to CNAv4\#, Cached Bindings to HoAv6* $\leftrightarrow$ CoAv6*, Source Port and Destination Port to the source port number and destination port number of the Agent Request message respectively. HoAv6*, CoAv6* and
HAAv6* can be acquired by adding a 96-bit NAT-PT prefix to HoAv4, CoAv4, HAAv4, while CNAv6 can be acquired by checking the NAT table, using CNAv4\# as the index. HoAv4, CoAv4, HAAv4 and CNAv4\# are carried in the Agent Request message. MIP-ALG sets the fields of HoTI/HoT, CoTI/CoT and State to 0 , and then sets the Lifetime to a value in which the creation of the entry must be accomplished.

(3) MIP-ALG generates HoTI message and CoTI message. The source address and destination address of HoTI message are HoAv6* and CNAv6, respectively, while the source address and destination address of CoTI message are CoAv6* and CNAv6, respectively. Both of these messages will be routed to CNv6.

(4) MIPv4/v6-TG intercepts HoT message and CoT message replied from CNv6, uses HoAv6* carried in these messages as an index to search MIP table, and finds this entry.

(5) For HoT message, MIP-ALG sets the HoTI/HoT field of the entry to 1 , while for CoT message, MIP-ALG sets the CoTI/CoT field of the entry to 1 . When both HoTI/HoT field and CoTI/CoT field become 1, MIP-ALG will generate a BU message, with CoAv6* and CNAv6 as its source address and destination address, respectively. This BU message will be routed to $\mathrm{CNv6.}$

(6) MIPv4/v6-TG intercepts BA message replied from CNv6. Then, MIPv4/v6-TG uses HoAv6* carried in the message as an index to search MIP table and finds this entry.

(7) MIP-ALG generates an Agent Reply message with CNAv4\# and CoAv4 as its source address and destination address, respectively. This message is sent through UDP. The source port and destination port are copied from the fields of Destination Port and Source Port, respectively. This Agent Reply message will be routed to MNv4.

(8) MIP-ALG sets the State of the entry to 1 (finished), and sets the Lifetime of the entry to the lifetime of the binding cache.

\section{B. The update of Type 001MIP Table Entries}

When MN moves within network of the same IP version and acquires a new care-of address, it will update the binding caches on HA and CN (when CN is in IPv6 network) as well as the binding caches on the related MIP table entry under some circumstances. Like the creation of MIP table entries, the update of the entry is triggered by MIP messages, such as Registration Request messages, BU messages, HoTI/HoT messages and CoTI/CoT messages. In the creation process, MIPv4/MIPv6 Message Entrance of the entry have been set. MIPv4/v6-TG can access a corresponding entry through these entrances when it intercepts a MIP message, and then updates the entry.

Note that when MN moves to a network of a different IP version, the original entry (if any) becomes invalid and a new MIP table entry should be created.

A Type 001 entry corresponds to a scenario where both HA and MN are located in IPv4 network, and CN is located in IPv6 network. According to RFC3344, MN does not register with CN. Therefore, MIPv4/v6-TG will not receive any 
Registration Request message sent by MN. The contents of the corresponding entry, except the Lifetime, do not need to be updated.

According to RFC3775, CN also maintains a binding cache which should be updated after $\mathrm{MN}$ acquires a new care-of address. In this scenario, however, the destination address of datagrams sent by CN consists of two parts: a 96-bit NAT-PT prefix and IPv4 care-of address. It is only based on the 96-bit NAT-PT prefix that the datagrams are routed to MIPv4/v6-TG. MIPv4/v6-TG will then send the datagrams to HA, rather than $\mathrm{MN}$. Therefore, as long as the binding cache on HA has been updated, the datagrams sent by $\mathrm{CN}$ will be correctly delivered to MN by HA.

\section{The usage of Type 001MIP Table Entries}

The introduction of MIP table aims to maintain MIP sessions in IPv4/v6 mixed networks. When a datagram sent by MN or CN passes through MIPv4/v6-TG, MIPv4/v6-TG will take out the destination address of the datagram and uses it as an index to search the MIP table. If a matching entry is found, MIPv4/v6-TG will process the datagram, based on the information recorded in the entry.

When an IPv4 datagram passes through MIPv4/v6-TG, MIPv4/v6-TG will take out its destination address and uses it as an index to search the MIP table. If a Type 001 entry is found, MIPv4/v6-TG will process the datagram as follows.

(1) NAT-PT, a component of MIPv4/v6-TG, translates the datagram into IPv6 format.

(2) MIP-ALG inserts a Destination Option extension header into the IPv6 datagram, and fills the header with the source address of the IPv6 datagram. Then MIP-ALG uses the source address as an index to search Cached Bindings field, and takes out the care-of address bound with the source address, and replaces the source address with the care-of address.

(3) MIPv4/v6-TG sends the processed IPv6 datagram.

When an IPv6 datagram passes through MIPv4/v6-TG, MIPv4/v6-TG takes out its destination address and uses it as an index to search the MIP table. If a Type 001 entry is found, MIPv4/v6-TG will process the datagram as follows.

1).MIPv4/v6-TG replaces the destination address of the datagram with the IPv6 home address, which is taken from the type 2 routing header of the datagram.

2).NAT-PT translates the datagram into IPv4 format.

3).MIPv4/v6-TG sends the processed IPv4 datagram.

\section{CONCLUSION}

Mobile IP is one of the trends of Internet techniques. Mobile IPv4 and Mobile IPv6 have been specified by IETF in RFC3344 and RFC3775 respectively. Recently the issue of transition from Mobile IPv4 to Mobile IPv6 has attracted much attention. Many suggestions, including some Internet Drafts, have been proposed on this issue. But none of them has been adopted as RFC by IETF.
The key to our solution is a MIP table, a newly introduced data structure. With the help of this gateway and the MIP table, RFC3344 and RFC3775 can be reused in IPv4 network and IPv6 network respectively. In this way, the Mobile IP entities in IPv4 network and the Mobile IP entities in IPv6 network can be transparent to each other.

Compared with other solutions, our solution has three main advantages. Firstly, it can work compatibly with RFC3344 and RFC3775. This is very important in that it makes Mobile IP in IPv4/v6 mixed networks possible without any update to the existing networks. Secondly, our solution introduces MIP table. We can use the MIP table to realize the communication in IPv4/v6 mixed network easily. Thirdly, the creation, usage and update of the MIP table entries is easily too. Through the simulation we find that the MIPv4/v6-TG can realize the basic communication in IPv4/v6 mixed network.

\section{REFERENCES}

[1] Charles E. Perkins, Editor, “IP Mobility Support for IPv4”, RFC3344, IETF, August 2002

[2] Charles E. Perkins, Editor, “Mobility Support in IPv6”, RFC3775, IETF, June 2004

[3] E. Nordmark, "Basic Transition Mechanisms for IPv6 Hosts and Routers”, RFC4219, IETF, October 2005

[4] A. Durand, “IPv6 Tunnel Broker”, RFC3053, IETF, January 2001

[5] E. Nordmark, "Stateless IP/ICMP Translation Algorithm (SIIT)", RFC2765, IETF, February 2000

[6] G. Tsirtsis, P. Srisuresh, "Network Address Translation-Protocol Translation (NAT-PT)”, RFC2776, IETF, February 2000

[7] Joo- Chul Lee, “ Considerations for Mobility Support in NAT-PT”, Internet Draft, IETF, June 2005 